\title{
DEFORMING CALABI-YAU ORBIFOLDS*
}

\author{
DOMINIC JOYCE ${ }^{\dagger}$
}

1. Introduction. A Calabi-Yau 3-fold is a compact complex 3-manifold $(X, J)$ equipped with a Ricci-flat Kähler metric $g$ and a holomorphic volume form $\Omega$ which is constant under the Levi-Civita connection of $g$. Suppose $X$ is a Calabi-Yau 3-fold and $G$ a finite group that acts on $X$ preserving $J, g$ and $\Omega$. Then $Z=X / G$ is a CalabiYau 3-orbifold. One can also construct Calabi-Yau 3-orbifolds $Z$ which are not of the form $X / G$, for instance as hypersurfaces in weighted projective spaces $\mathbb{C P}_{a_{0}, \ldots, a_{4}}^{4}$ as in Candelas et al. [2].

Often it is possible to find a compact manifold $Y$ that desingularizes $Z$, and carries a family of Calabi-Yau structures that converge to the singular Calabi-Yau structure on $Z$ in a well-defined sense, so that the orbifold metric on $Z$ may be regarded as the degenerate case in a smooth family of Calabi-Yau metrics on $Y$.

The two main strategies for desingularizing $Z$ to get $Y$ are called resolution and deformation. A resolution $(Y, \pi)$ of $Z$ is called a crepant resolution if $K_{Y} \cong \pi^{*}\left(K_{Z}\right)$. To get a Calabi-Yau structure on $Y$ we must choose a crepant resolution. A nonsingular deformation of $Z$ is called a smoothing.

We can also combine the two processes by taking a crepant resolution of a singular deformation of $Z$, which we will call a $C R$-deformation of $Z$. Thus, crepant resolutions, smoothings and CR-deformations are three different ways to desingularize a Calabi-Yau orbifold $Z$ to get a new Calabi-Yau 3-fold $Y$. As a shorthand we will sometimes use desingularize and desingularization to mean either a crepant resolution, or a smoothing, or a CR-deformation.

Much is already known about the crepant resolutions of $Z$. For example, $Z$ always admits at least one crepant resolution, distinct crepant resolutions of $Z$ are related by 'flops', and the Hodge numbers of all crepant resolutions of $Z$ are the same, and can be written down in terms of $X$ and $G$. For more information on this, see for instance Reid [9], Ito and Reid [3] and Roan [10]. However, the topology of smoothings and CR-deformations of $Z$ is less well understood.

In this paper we describe a topological mechanism through which a Calabi-Yau orbifold $Z$ with singularities of codimension two may admit a number of topologically distinct resolutions, smoothings and CR-deformations, with a variety of Betti numbers. This mechanism is related to the Weyl group of the codimension two singularities, and we refer to it as twisting by the Weyl group.

We illustrate our ideas upon two orbifolds. The first example $T^{6} / \mathbb{Z}_{4}$ has crepant resolutions and smoothings with 5 different sets of Betti numbers, which are easily described. The second example $T^{6} / \mathbb{Z}_{2}^{2}$ is much more complicated. There are a very large number of ways of desingularizing it, which we are unable to classify completely, realizing many different sets of Betti numbers. It seems surprising that such a simple orbifold can have crepant resolutions, smoothings and CR-deformations giving CalabiYau 3-folds in so many different ways.

We now explain the idea of 'twisting by the Weyl group'. Suppose $G$ is a finite subgroup of $S U(3)$ with a normal subgroup $H$ contained in some $S U(2) \subset S U(3)$, but where $G$ is not itself contained in $S U(2)$. Then $\mathbb{C}^{3} / H \cong \mathbb{C} \times \mathbb{C}^{2} / H$, and $G / H$ is

*Received June 25, 1999; accepted for publication March 17, 2000.

${ }^{\dagger}$ Lincoln College, Oxford, OX1 3DR, England (dominic.joyce@lincoln.ox.ac.uk). 
a finite cyclic group $\mathbb{Z}_{k}$ for some $k>1$. To construct a crepant resolution, smoothing or CR-deformation $Y$ of $\mathbb{C}^{3} / G$, we may proceed in two stages.

The first stage is to choose a desingularization $X$ of $\mathbb{C}^{2} / H$, so that $\mathbb{C} \times X$ is a crepant resolution, smoothing or CR-deformation of $\mathbb{C}^{3} / H$. Then we hope to find an action of $\mathbb{Z}_{k}=G / H$ upon $\mathbb{C} \times X$, which is asymptotic to the prescribed action of $\mathbb{Z}_{k}$ on $\mathbb{C}^{3} / H$. If there exists such an action, then the second stage is to make $Y$ as a crepant resolution, smoothing or CR-deformation of $(\mathbb{C} \times X) / \mathbb{Z}_{k}$.

It is well known that if $H \subset S U(2)$ is a finite subgroup then $\mathbb{C}^{2} / H$ admits a unique crepant resolution $X^{\prime}$, and that any smoothing or CR-deformation $X$ of $\mathbb{C}^{2} / H$ is diffeomorphic to $X^{\prime}$. Thus, the diffeomorphism type of $X$ is fixed uniquely by $H$. However, our key observation is that the action of $\mathbb{Z}_{k}$ on $X$ and on its cohomology is not always uniquely determined by $G$.

Instead, there can be a finite number of topologically distinct ways for $\mathbb{Z}_{k}$ to act on $X$, depending on the choice of complex structure of $X$, and on the level of cohomology these actions differ by an element of the Weyl group of the singularity $\mathbb{C}^{2} / H$. For one of these $\mathbb{Z}_{k}$-actions the desingularization $Y$ of $(\mathbb{C} \times X) / \mathbb{Z}_{k}$ has the topology of a crepant resolution, but for other choices of the $\mathbb{Z}_{k}$-action $Y$ does not have this topology.

As the idea of making Calabi-Yau 3-folds out of torus orbifolds $T^{6} / G$ goes back a long way, this paper naturally has similarities with the work of other authors. The first paper to construct Calabi-Yau 3-folds using crepant resolutions of $T^{6} / G$ was Roan and Yau [11], and was followed by many others.

The method of constructing Calabi-Yau 3-folds by deforming singular Calabi-Yau 3 -folds has also been explored by a number of authors - see for instance Tian [14] for an important result on deformations of Calabi-Yau 3-folds with ordinary double points.

However, as far as the author can tell, there are few papers which combine these ideas, and construct Calabi-Yau 3-folds by deforming orbifolds $T^{6} / G$ with codimension two singularities. One such paper is Vafa and Witten $[15, \S 2]$, who construct a Calabi-Yau 3-fold by smoothing $T^{6} / \mathbb{Z}_{2}^{2}$.

The two main ideas in this paper which the author hopes are new are the use of Weyl groups of codimension two singularities to find and describe topologically distinct ways of making Calabi-Yau 3-folds out of orbifolds by CR-deformations, and the fact that just one orbifold can have a surprisingly large number of Calabi-Yau desingularizations, realizing many different sets of Betti numbers.

The rest of the paper is set out as follows. Section 2 summarizes the theory of singularities $\mathbb{C}^{2} / H$ for $H$ a finite subgroup of $S U(2)$, their resolutions and deformations, and the idea of the Weyl group. Then $\S 3$ explains some theory about the orbifolds we are interested in, and the topology of their crepant resolutions, smoothings and CR-deformations. Sections 4 and 5 apply these ideas to the orbifolds $T^{6} / \mathbb{Z}_{4}$ and $T^{6} / \mathbb{Z}_{2}^{2}$.

2. Kleinian singularities and ALE spaces. The quotient singularities $\mathbb{C}^{2} / H$, for $H$ a finite subgroup of $S U(2)$, were first classified by Klein in 1884 and are called Kleinian singularities; they are also called $D u$ Val surface singularities, or rational double points. The theory of these singularities and their resolutions is very rich, and has many connections to other areas of mathematics. Most of the following facts are taken from McKay [8], Slodowy [13], and Kronheimer [6, 7].

There is a 1-1 correspondence between finite subgroups $H \subset S U(2)$ and the Dynkin diagrams of type $A_{r}(r \geq 0), D_{r}(r \geq 4), E_{6}, E_{7}$ and $E_{8}$. Let $\Gamma$ be the Dynkin 
diagram associated to $H$. These Dynkin diagrams appear in the classification of Lie groups, and each one corresponds to a unique compact, simple Lie group; they are the set of such diagrams containing no double or triple edges.

Each singularity $\mathbb{C}^{2} / H$ admits a unique crepant resolution $(X, \pi)$. The preimage $\pi^{-1}(0)$ of the singular point is a union of a finite number of rational curves in $X$. These curves correspond naturally to the vertices of $\Gamma$. They all have self-intersection -2 , and two curves intersect transversely at one point if and only if the corresponding vertices are joined by an edge in the diagram; otherwise the curves do not intersect.

These curves give a basis for the homology group $H_{2}(X, \mathbb{Z})$, which may be identified with the root lattice of the diagram, and the intersection form with respect to this basis is the negative of the Cartan matrix of $\Gamma$. Define $\Delta$ to be $\left\{\delta \in H_{2}(X, \mathbb{Z})\right.$ : $\delta \cdot \delta=-2\}$. Then $\Delta$ is identified with the set of roots of the diagram. There are also 1-1 correspondences between the curves and the nonidentity conjugacy classes in $H$, and also the nontrivial representations of $H$; it makes sense to regard the nonidentity conjugacy classes as a basis for $H_{2}(X, \mathbb{Z})$, and the nontrivial representations as a basis for $H^{2}(X, \mathbb{Z})$.

By the theory of Lie groups, the Dynkin diagram $\Gamma$ of $\mathbb{C}^{2} / H$ has a Weyl group $W$, and a representation of $W$ on the root lattice $H_{2}(X, \mathbb{Z})$ of $\Gamma$. This action of $W$ preserves the subset $\Delta$ and the intersection form on $H_{2}(X, \mathbb{Z})$, and by duality $W$ also acts on $H^{2}(X, \mathbb{Z})$. Let $\operatorname{Aut}(\Gamma)$ be the group of automorphisms of the graph $\Gamma$, which is given by

$$
\operatorname{Aut}(\Gamma)= \begin{cases}\{1\} & \text { if } \Gamma=A_{1}, E_{7} \text { or } E_{8} \\ \mathbb{Z}_{2} & \text { if } \Gamma=A_{k}(k \geq 2), D_{k}(k \geq 5) \text { or } E_{6}, \\ S_{3} & \text { if } \Gamma=D_{4}\end{cases}
$$

Now the vertices of $\Gamma$ correspond to the basis elements of $H_{2}(X, \mathbb{Z})$, so that Aut $(\Gamma)$ acts naturally on $H_{2}(X, \mathbb{Z})$, preserving the intersection form. But the Weyl group $W$ also acts on $H_{2}(X, \mathbb{Z})$. It turns out that there is a natural semidirect product $\operatorname{Aut}(\Gamma) \ltimes W$, and the actions of $\operatorname{Aut}(\Gamma)$ and $W$ on $H_{2}(X, \mathbb{Z})$ combine to give a representation of $\operatorname{Aut}(\Gamma) \ltimes W$ on $H_{2}(X, \mathbb{Z})$. Define $\rho$ to be the dual representation of $\operatorname{Aut}(\Gamma) \ltimes W$ on both $H^{2}(X, \mathbb{R})$ and $H^{2}(X, \mathbb{C})$. The action of each element of $\operatorname{Aut}(\Gamma) \ltimes W$ is induced by a diffeomorphism of $X$, so we can interpret $\operatorname{Aut}(\Gamma) \ltimes W$ as a group of isotopy classes of diffeomorphisms of $X$. However, in general Aut $(\Gamma) \ltimes W$ is not a group of diffeomorphisms of $X$, nor an isometry group of any of the metrics or complex structures on $X$.

The singularities $\mathbb{C}^{2} / H$ can be desingularized by deformation as well as by crepant resolution. Klein found that each singularity $\mathbb{C}^{2} / H$ is isomorphic as an affine complex variety to the zeros of a polynomial on $\mathbb{C}^{3}$. For example, $\mathbb{C}^{2} / \mathbb{Z}_{k}$ may be identified with the set of $(x, y, z) \in \mathbb{C}^{3}$ with $x y-z^{k}=0$. The deformations of $\mathbb{C}^{2} / H$ are constructed by adding terms of lower order in $x, y$ and $z$ to this polynomial. All of the smooth deformations of $\mathbb{C}^{2} / H$ are diffeomorphic to the unique crepant resolution $X$ of $\mathbb{C}^{2} / H$.

Each of these desingularizations of $\mathbb{C}^{2} / H$ carries a special family of metrics with holonomy $S U(2)$. The metrics are asymptotic up to $O\left(r^{-4}\right)$ to the Euclidean metric on $\mathbb{C}^{2} / H$, and so are called Asymptotically Locally Euclidean; the complex manifold $X$ with its Kähler metric is called an ALE space. A complete construction and classification of ALE spaces was carried out by Kronheimer [6, 7], and we describe it next.

Let $Y$ be an ALE space asymptotic to $\mathbb{C}^{2} / H$. Then $Y$ is diffeomorphic to $X$, and carries a geometric structure which is encoded in the Kähler form $\omega$ and the 
holomorphic volume form $\Omega$ of $X$. Both $\omega$ and $\Omega$ are closed forms, so they define de Rham cohomology classes $\alpha=[\omega] \in H^{2}(X, \mathbb{R})$ and $\beta=[\Omega] \in H^{2}(X, \mathbb{C})$. Thus, to each ALE space $Y$ we may associate the pair $(\alpha, \beta) \in H^{2}(X, \mathbb{R}) \times H^{2}(X, \mathbb{C})$.

For each pair $(\alpha, \beta) \in H^{2}(X, \mathbb{R}) \times H^{2}(X, \mathbb{C})$, Kronheimer [6] defined an explicit, possibly singular ALE space $X_{\alpha, \beta}$ asymptotic to $\mathbb{C}^{2} / H$, using the hyperkähler quotient construction. Let $U$ be the subset

$$
U=\left\{(\alpha, \beta) \in H^{2}(X, \mathbb{R}) \times H^{2}(X, \mathbb{C}): \alpha(\delta) \neq 0 \text { or } \beta(\delta) \neq 0 \text { for all } \delta \in \Delta\right\} .
$$

Then $U$ is a dense open subset of $H^{2}(X, \mathbb{R}) \times H^{2}(X, \mathbb{C})$. Kronheimer showed that if $(\alpha, \beta) \notin U$ then $X_{\alpha, \beta}$ is an orbifold, and if $(\alpha, \beta) \in U$ then $X_{\alpha, \beta}$ is nonsingular and diffeomorphic to $X$, and the Kähler form $\omega$ and holomorphic volume form $\Omega$ of $X_{\alpha, \beta}$ have cohomology classes $[\omega]=\alpha$ and $[\Omega]=\beta$. The manifolds $X_{\alpha, \beta}$ for $(\alpha, \beta) \in U$ form a family diffeomorphic to $X \times U$.

Next, Kronheimer [7] showed that if $Y$ is any ALE space asymptotic to $\mathbb{C}^{2} / H$ then the associated pair $(\alpha, \beta)$ must lie in $U$, and that if $Y_{1}$ and $Y_{2}$ are two ALE spaces asymptotic to $\mathbb{C}^{2} / H$ that both yield the same pair $(\alpha, \beta)$, then $Y_{1}, Y_{2}$ are isomorphic as ALE spaces. Combining these results we see that every ALE space $Y$ asymptotic to $\mathbb{C}^{2} / H$ is isomorphic to $X_{\alpha, \beta}$ for some pair $(\alpha, \beta) \in U$, so we have a complete description of all ALE spaces.

The group $\operatorname{Aut}(\Gamma) \ltimes W$ associated to $\mathbb{C}^{2} / H$ acts on Kronheimer's construction, in the following way. The obvious action of $\operatorname{Aut}(\Gamma) \ltimes W$ on $H^{2}(X, \mathbb{R}) \times H^{2}(X, \mathbb{C})$ preserves the subset $U$, so that $\operatorname{Aut}(\Gamma) \ltimes W$ also acts on $U$. The action extends naturally to the hyperkähler quotient construction that Kronheimer uses to construct $X_{\alpha, \beta}$, and this shows that if $w \in \operatorname{Aut}(\Gamma) \ltimes W$ and $(\alpha, \beta) \in U$, then $X_{w \cdot \alpha, w \cdot \beta}$ is isomorphic to $X_{\alpha, \beta}$ as an ALE space.

Moreover, if $w \in W$ rather than $\operatorname{Aut}(\Gamma) \ltimes W$, then there is a unique ALE space isomorphism between $X_{w \cdot \alpha, w \cdot \beta}$ and $X_{\alpha, \beta}$ that is asymptotic to the identity at infinity. One can also show that if $X_{\alpha^{\prime}, \beta^{\prime}}$ is isomorphic to $X_{\alpha, \beta}$ as an ALE space, then $\left(\alpha^{\prime}, \beta^{\prime}\right)=$ $(w \cdot \alpha, w \cdot \beta)$ for some $w \in \operatorname{Aut}(\Gamma) \ltimes W$. If in addition the isomorphism between $X_{\alpha^{\prime}, \beta^{\prime}}$ and $X_{\alpha, \beta}$ is asymptotic to the identity at infinity, then $w \in W$.

Let $w \in \operatorname{Aut}(\Gamma) \ltimes W$. Now $X_{\alpha, \beta}$ and $X_{w \cdot \alpha, w \cdot \beta}$ are both diffeomorphic to $X$, under diffeomorphisms that are natural up to isotopy. The identification between $X_{\alpha, \beta}$ and $X_{w \cdot \alpha, w \cdot \beta}$ that comes from their isomorphism as ALE spaces can thus be thought of as a diffeomorphism of $X$, up to isotopy. The corresponding isotopy class of diffeomorphisms of $X$ is identified with $w \in \operatorname{Aut}(\Gamma) \ltimes W$, regarding $\operatorname{Aut}(\Gamma) \ltimes W$ as a group of isotopy classes of diffeomorphisms of $X$, as above. In particular, the identification between $X_{\alpha, \beta}$ and $X_{w \cdot \alpha, w \cdot \beta}$ induces the action of $w$ on $H^{2}(X, \mathbb{R})$ and $H^{2}(X, \mathbb{C})$, and this is why it is possible for two isomorphic Kähler forms apparently to have two different cohomology classes $\alpha$ and $w \cdot \alpha$.

Here is a heuristic description of what is going on. When we desingularize $\mathbb{C}^{2} / H$ we replace the singular point by a bunch of 2 -spheres, and this introduces nontrivial homology classes in $H_{2}(X, \mathbb{Z})$. The Weyl group $W$ then acts as a kind of 'internal symmetry group' on the new homology classes; we can visualize elements of $W$ as diffeomorphisms of $X$ that are the identity outside a small neighbourhood of the 2 -spheres. Elements of $\operatorname{Aut}(\Gamma)$ also act as diffeomorphisms of $X$, but they act nontrivially near infinity.

These diffeomorphisms of $X$ can in fact be described as generalized Dehn twists of $X$, and the Weyl group $W$ is the group of isotopy classes of diffeomorphisms of $X$ generated by Dehn twists along the exceptional 2-spheres. For explicit descriptions of 
the generalized Dehn twist of $T^{*} \mathcal{S}^{2}$ along the zero section, which is the case $H=\mathbb{Z}_{2}$ above, see Arnold [1, §2] and Seidel [12].

The ALE spaces $X_{\alpha, \beta}$ for $(\alpha, \beta) \in U$ can be thought of as a family of Kähler structures upon the fixed real 4-manifold $X$. Then $X_{\alpha, \beta}$ and $X_{w \cdot \alpha, w \cdot \beta}$ represent Kähler structures on $X$ that are equivalent under a diffeomorphism $\phi$ of $X$ corresponding to $w$. If $w \neq 1$ then $\phi$ is not isotopic to the identity, and acts nontrivially on $H^{2}(X, \mathbb{R})$ and $H^{2}(X, \mathbb{C})$.

3. Desingularizing Calabi-Yau orbifolds. We are interested in desingularizing Calabi-Yau orbifolds of dimension 3 whose singularities are modelled upon $\mathbb{C}^{3} / G$, where $G$ is a finite subgroup of $S U(3)$, and $\mathbb{C}^{3} / G$ has singularities in codimension two. If we first understand the different ways of resolving, smoothing and CR-deforming such $\mathbb{C}^{3} / G$, this will give us a local model for how to desingularize more general Calabi-Yau orbifolds $Z$.

The usual point of view is that one constructs a CR-deformation $Y$ of $Z$ using complex geometry, and then applies Yau's theorem to show that there exist CalabiYau metrics on $Y$. Thus, the complex structure of $Y$ is close to that of $Z$ in some sense, but we don't actually know that the Calabi-Yau metric on $Y$ is close to the orbifold metric on $Z$. Now the author conjectures that this should be so.

That is, given a family $\left\{Y_{t}: t \in(-\epsilon, \epsilon)\right\}$ of CR-deformations of $Z$ with $Y_{0}=Z$, the author believes that there should exist a corresponding family of Calabi-Yau metrics $g_{t}$ on $Y_{t}$ which converge to the orbifold metric on $Z$ as $t \rightarrow 0$, say in the GromovHausdorff sense. Moreover, for small $t$ and near the desingularization of an orbifold point in $Z$ modelled on $\mathbb{C}^{3} / G$, the metric $g_{t}$ should be close to a Quasi-ALE metric with holonomy in $S U(3)$ on a CR-deformation of $\mathbb{C}^{3} / G$, in the sense of [4].

We shall not actually use this conjecture in the paper. However, it motivates the approach we take, which is based on the idea that in studying the different CRdeformations $X$ of $\mathbb{C}^{3} / G$, it is important to consider and describe the appropriate Quasi-ALE metrics on $X$ as well as the complex structures. In particular, the properties of Kronheimer's metrics on ALE spaces described in $\S 2$ will be pivotal to our constructions.

Suppose $G \subset S U(3)$ is finite and $\mathbb{C}^{3} / G$ has codimension two singularities. Pick $x \in \mathbb{C}^{3}$ such that $x G$ is a generic point in the codimension two singular set, and let $H$ be $\{h \in G: h(x)=x\}$, the stabilizer subgroup of $x$ in $G$. Then there is a natural orthogonal splitting $\mathbb{C}^{3}=\mathbb{C} \oplus \mathbb{C}^{2}$, such that $x \neq 0$ lies in $\mathbb{C}$, and $H$ fixes $\mathbb{C}$ and acts on $\mathbb{C}^{2}$ as a finite, nontrivial subgroup of $S U(2)$. We may write $\mathbb{C}^{3} / H=\mathbb{C} \times\left(\mathbb{C}^{2} / H\right)$, where $\mathbb{C}^{2} / H$ is one of the Kleinian singularities of $\S 2$.

We shall restrict our attention to the case that $H$ is a normal subgroup of $G$. If $H$ is not normal then things are more difficult. So suppose that $H$ is normal in $G$, and let $K$ be the quotient group $G / H$. Then $K$ acts naturally on $\mathbb{C} \times \mathbb{C}^{2} / H$, and $\left(\mathbb{C} \times \mathbb{C}^{2} / H\right) / K=\mathbb{C}^{3} / G$. Let the notation $X, \Gamma, \Delta, W, \operatorname{Aut}(\Gamma), \rho, U$ and $X_{\alpha, \beta}$ all be as defined in the previous section.

We begin by constructing two natural group homomorphisms $\phi: K \rightarrow U(1)$ and $\psi: K \rightarrow \operatorname{Aut}(\Gamma)$. Since $H$ is the subgroup of $G$ fixing $\mathbb{C}$ and is normal in $G$, it follows that $G$ preserves the splitting $\mathbb{C}^{3}=\mathbb{C} \oplus \mathbb{C}^{2}$. Therefore $G$ is a subgroup of $S(U(1) \times U(2))$, the subgroup of $S U(3)$ preserving this splitting, and we may write each element $g \in G$ as a pair $(\sigma, \tau)$, where $\sigma \in U(1), \tau \in U(2)$, and $\sigma \cdot \operatorname{det} \tau=1$. Then $g \in H$ if and only if $\sigma=1 \in U(1)$. Define a map $\phi: K \rightarrow U(1)$ by $\phi(g H)=\sigma$ for each $g \in G$, where $g=(\sigma, \tau)$. It is easy to see that $\phi$ is well-defined, and a group homomorphism. 
Write $C_{h}$ for the conjugacy class of $h$ in $H$, and let $S_{H}$ be the set of nonidentity conjugacy classes in $H$. As $H$ is a normal subgroup, $g C_{h} g^{-1}$ is also a conjugacy class in $H$ for each $g \in G$, which is the identity if and only if $C_{h}$ is. Thus $C_{h} \mapsto g C_{h} g^{-1}$ defines a map from $S_{H}$ to itself. Define a map from $K \times S_{H}$ to $S_{H}$ by $\left(g H, C_{h}\right) \mapsto$ $g C_{h} g^{-1}$. Then this map is well-defined and is an action of $K$ on $S_{H}$. But there is a natural correspondence between $S_{H}$, the nonidentity conjugacy classes in $H$, and the vertices of the Dynkin diagram $\Gamma$. Thus $K$ acts on the vertices of $\Gamma$. In fact $K$ acts by automorphisms of the whole graph, and this defines the group homomorphism $\psi: K \rightarrow \operatorname{Aut}(\Gamma)$ that we want.

Now let $(\alpha, \beta) \in U$, so that $X_{\alpha, \beta}$ is a nonsingular ALE space, diffeomorphic to $X$, and asymptotic to $\mathbb{C}^{2} / H$ as in $\S 2$. Then $\mathbb{C} \times X_{\alpha, \beta}$ desingularizes $\mathbb{C} \times \mathbb{C}^{2} / H$, and has a natural Calabi-Yau structure. Our goal is to choose $(\alpha, \beta)$ such that $\mathbb{C} \times X_{\alpha, \beta}$ admits a $K$-action preserving this Calabi-Yau structure, which is asymptotic to the natural action of $K$ on $\mathbb{C} \times \mathbb{C}^{2} / H$. To achieve this, we must work out what conditions $\alpha$ and $\beta$ must satisfy for such a $K$-action to exist.

First consider how $K$ can act on $X$, as a group of diffeomorphisms. The action of $K$ on $\mathbb{C}^{2} / H$ only determines how $K$ should act 'near infinity' in $X$. But elements of $W$ may be visualized as diffeomorphisms of $X$ that are the identity near infinity. Thus the action of $K$ on $X$ may not be uniquely determined by the asymptotic conditions on it, but instead there may be several such actions, differing only by elements of $W$. The data we need to determine how $K$ acts on $X$ is a group homomorphism $\chi: K \rightarrow \operatorname{Aut}(\Gamma) \ltimes W$, such that $\pi \circ \chi=\psi$, where $\pi: \operatorname{Aut}(\Gamma) \ltimes W \rightarrow \operatorname{Aut}(\Gamma)$ is the natural projection, so that $\chi$ lifts $\psi$ from $\operatorname{Aut}(\Gamma)$ to $\operatorname{Aut}(\Gamma) \ltimes W$. Choose such a homomorphism $\chi$.

There always exists at least one such homomorphism, because there is a canonical choice for $\chi$, which will yield crepant resolutions of $\mathbb{C}^{3} / G$ in the construction below. As $\operatorname{Aut}(\Gamma)$ is naturally isomorphic to a subgroup of $\operatorname{Aut}(\Gamma) \ltimes W$, we can regard $\psi: K \rightarrow \operatorname{Aut}(\Gamma)$ as a homomorphism $K \rightarrow \operatorname{Aut}(\Gamma) \ltimes W$, and this is the canonical choice for $\chi$. However, for many groups $G, H$ there are other, different choices for $\chi$, and we may be able to use these to construct smoothings or CR-deformations of $\mathbb{C}^{3} / G$ which do not have the topology of a crepant resolution.

Since $\chi: K \rightarrow \operatorname{Aut}\left(\Gamma^{\prime}\right) \ltimes W$ is a group homomorphism and $\rho$ is a representation of $\operatorname{Aut}(\Gamma) \ltimes W$ on $H^{2}(X, \mathbb{R})$ and $H^{2}(X, \mathbb{C})$, we see that $\rho \circ \chi$ is a representation of $K$ on $H^{2}(X, \mathbb{R})$ and $H^{2}(X, \mathbb{C})$. So, suppose for the moment that $K$ acts on $X$ as a group of diffeomorphisms, with action asymptotic to the prescribed action of $K$ on $\mathbb{C}^{2} / H$, such that the induced action of $K$ on $H^{2}(X, \mathbb{R})$ and $H^{2}(X, \mathbb{C})$ is $\rho \circ \chi$.

Next, we choose $(\alpha, \beta) \in U$ and identify $X_{\alpha, \beta}$ with $X$ as a real 4-manifold, so that $K$ acts on $X_{\alpha, \beta}$, and so on $\mathbb{C} \times X_{\alpha, \beta}$. What is the condition on the pair $(\alpha, \beta)$ for this $K$-action to preserve the natural Calabi-Yau structure on $\mathbb{C} \times X_{\alpha, \beta}$ ? Let the Kähler form and holomorphic volume form of $\mathbb{C}$ be $\omega$ and $\Omega$, and let the Kähler form and holomorphic volume form of $X_{\alpha, \beta}$ be $\omega^{\prime}$ and $\Omega^{\prime}$, respectively. Then the Kähler form of $\mathbb{C} \times X_{\alpha, \beta}$ is $\omega+\omega^{\prime}$, and the holomorphic volume form of $\mathbb{C} \times X_{\alpha, \beta}$ is $\Omega \wedge \Omega^{\prime}$. Thus the $K$-action on $\mathbb{C} \times X_{\alpha, \beta}$ must preserve both $\omega+\omega^{\prime}$ and $\Omega \wedge \Omega^{\prime}$.

Write $g \in G$ as a pair $(\sigma, \tau)$ as above, where $\sigma \in U(1)$ and $\tau \in U(2)$. Then $g H \in K$ acts on $\omega$ and $\Omega$ by $g H \cdot \omega=\omega$ and $g H \cdot \Omega=\sigma \cdot \Omega$. Therefore, the Calabi-Yau structure of $\mathbb{C} \times X_{\alpha, \beta}$ is $K$-invariant if $g H \cdot \omega^{\prime}=\omega^{\prime}$ and $\sigma \cdot\left(g H \cdot \Omega^{\prime}\right)=\Omega^{\prime}$. Now the cohomology classes of $\omega^{\prime}$ and $\Omega^{\prime}$ are $\alpha$ and $\beta$ respectively, and $\sigma=\phi(g H)$ from above. Therefore a necessary condition on the pair $(\alpha, \beta)$ for the Calabi-Yau structure on 
$\mathbb{C} \times X_{\alpha, \beta}$ to be $K$-invariant is

$$
\rho \circ \chi(g H) \alpha=\alpha \quad \text { and } \quad \phi(g H) \cdot \rho \circ \chi(g H) \beta=\beta \quad \text { for all } g H \in K .
$$

It turns out that equation (3.1) is also a sufficient condition for there to exist a $K$-action on $X_{\alpha, \beta}$ with all the properties we require. In particular, we do not need the assumption we made above about the existence of a suitable action of $K$ on $X$ by diffeomorphisms, because equation (3.1) guarantees this. We now explain why (3.1) is a sufficient condition. Recall from $\S 2$ that if $w \in \operatorname{Aut}(\Gamma) \ltimes W$ and $(\alpha, \beta) \in U$ then $X_{\alpha, \beta}$ and $X_{w \cdot \alpha, w \cdot \beta}$ are isomorphic as ALE spaces, and that if $w \in W$ then there is a unique isomorphism which is asymptotic to the identity at infinity.

Extending the arguments used to show this, one can prove the following result. Let $g H \in K$ and $(\alpha, \beta)$ and $\left(\alpha^{\prime}, \beta^{\prime}\right)$ lie in $U$, and consider a map $\Theta_{g H}: X_{\alpha, \beta} \rightarrow X_{\alpha^{\prime}, \beta^{\prime}}$ that is an isomorphism of Kähler manifolds, is asymptotic to the action of $g H$ on $\mathbb{C}^{2} / H$, multiplies holomorphic volume forms by $\phi(g H)^{-1}$, and acts on cohomology by $\rho \circ \chi(g H)$. Then the necessary and sufficient condition for there to exist such a map $\Theta_{g H}$ is that $\rho \circ \chi(g H) \alpha=\alpha^{\prime}$ and $\phi(g H) \cdot \rho \circ \chi(g H) \beta=\beta^{\prime}$, and if it exists then $\Theta_{g H}$ is unique.

But any solution $(\alpha, \beta)$ to $(3.1)$ satisfies these conditions with $\left(\alpha^{\prime}, \beta^{\prime}\right)=(\alpha, \beta)$. Therefore, this result guarantees the existence and uniqueness of a map $\Theta_{g H}$ from $X_{\alpha, \beta}$ to itself with the properties above, for each $g H \in K$. It is then easy to show that the maps $\Theta_{g H}$ yield an action of $K$ on $X_{\alpha, \beta}$ with all the properties we need. We summarize our progress so far in the following theorem; the final part is left as an exercise for the reader.

THEOREM 3.1. Using the above notation, suppose that $\chi: K \rightarrow \operatorname{Aut}(\Gamma) \ltimes W$ is a group homomorphism such that $\pi \circ \chi=\psi$, and suppose that $(\alpha, \beta) \in U$ satisfies the condition that $\rho \circ \chi(g H) \alpha=\alpha$ and $\phi(g H) \cdot \rho \circ \chi(g H) \beta=\beta$ for all $g H \in K$. Then there exists a unique action of $K$ on $\mathbb{C} \times X_{\alpha, \beta}$ that preserves the Calabi-Yau structure of $\mathbb{C} \times X_{\alpha, \beta}$ and is asymptotic to the natural action of $K$ on $\mathbb{C} \times \mathbb{C}^{2} / H$. The representation of $K$ on $H^{2}\left(X_{\alpha, \beta}, \mathbb{R}\right)$ induced by this action is $\rho \circ \chi$, identifying $X_{\alpha, \beta}$ and $X$ as real 4-manifolds. Moreover, if $Y$ is any ALE space asymptotic to $\mathbb{C}^{2} / H$ such that $\mathbb{C} \times Y$ admits a $K$-action preserving the Calabi-Yau structure and asymptotic to the given action on $\mathbb{C} \times \mathbb{C}^{2} / H$, then $Y$ arises from this construction.

Let us now consider the condition (3.1) more closely. What it really means is that $\alpha$ has to be invariant under the action $\rho \circ \chi$ of $K$ on $H^{2}(X, \mathbb{R})$, but $\beta$ has to be invariant under the action $\phi \cdot \rho \circ \chi$ of $K$ on $H^{2}(X, \mathbb{C})$. When $\phi$ is nontrivial, these two $K$-actions are different, and will have different invariant subspaces.

Our construction only works if we are able to choose $(\alpha, \beta) \in U$ satisfying (3.1). Thus by definition of $U$, we must find $K$-invariant elements $\alpha$ and $\beta$ such that either $\alpha(\delta) \neq 0$ or $\beta(\delta) \neq 0$ for each $\delta \in \Delta$. To satisfy this condition in examples, the fact that the two $K$-actions are different is important. For instance, it can happen that for some $\delta \in \Delta$, every element $\alpha \in H^{2}(X, \mathbb{R})$ invariant under the $K$-action $\rho \circ \chi$ satisfies $\alpha(\delta)=0$. But because $\beta$ must be invariant under a different $K$-action $\phi \cdot \rho \circ \chi$, there may still exist a suitable element $\beta$ with $\beta(\delta) \neq 0$.

The goal of this section is to find crepant resolutions, smoothings and CRdeformations of $\mathbb{C}^{3} / G$. We divide the problem into two stages, firstly to desingularize $\mathbb{C}^{3} / H$ to get $\mathbb{C} \times X_{\alpha, \beta}$ with a $K$-action, and then secondly to divide by $K$ and desingularize the result $\left(\mathbb{C} \times X_{\alpha, \beta}\right) / K$. So far we have discussed only the first stage in this process. But what about the second stage?

In fact, at the second stage three things can happen. Firstly, the $K$-action on $X_{\alpha, \beta}$ may have no fixed points. In this case $\left(\mathbb{C} \times X_{\alpha, \beta}\right) / K$ has no singularities, 
and is itself a smoothing or CR-deformation of $\mathbb{C}^{3} / G$. Secondly, the singularities of $\left(\mathbb{C} \times X_{\alpha, \beta}\right) / K$ may be isolated points. In this case we must take a crepant resolution. And thirdly, $\left(\mathbb{C} \times X_{\alpha, \beta}\right) / K$ may have singularities in codimension 2 . In this case we are free to apply the method above again.

That is, the singularities of $\left(\mathbb{C} \times X_{\alpha, \beta}\right) / K$ are locally modelled on $\mathbb{C}^{3} / G^{\prime}$, where $G^{\prime}$ is a finite subgroup of $S U(3)$ that is isomorphic to a subgroup of $K$. As the singularities have codimension 2, there is a subgroup $H^{\prime}$ in $G^{\prime}$ contained in some $S U(2) \subset S U(3)$. We may use the method above to find ways to desingularize $\mathbb{C}^{3} / G^{\prime}$, and use these as a local model to desingularize $\left(\mathbb{C} \times X_{\alpha, \beta}\right) / K$. Note that $\left|G^{\prime}\right| \leq|K|$ as $G^{\prime}$ is a subgroup of $K$, and $|K|=|G| /|H|<|G|$, so that $\left|G^{\prime}\right|<|G|$. Thus, if we use the method iteratively the size of the quotient groups decreases at each stage, and the process must terminate.

4. An example. We now apply the theory of the previous section to the example of $\mathbb{C}^{3} / \mathbb{Z}_{4}$. First in $\S 4.1$ we show that $\mathbb{C}^{3} / \mathbb{Z}_{4}$ has a crepant resolution and a smoothing, which are topologically distinct. Then in $\S 4.2$ we use this to desingularize a compact Calabi-Yau orbifold $T^{6} / \mathbb{Z}_{4}$.

4.1. Crepant resolutions and smoothings of $\mathbb{C}^{3} / \mathbb{Z}_{4}$. Let $\mathbb{C}^{3}$ have complex coordinates $\left(z_{1}, z_{2}, z_{3}\right)$, and define $\kappa: \mathbb{C}^{3} \rightarrow \mathbb{C}^{3}$ by

$$
\kappa:\left(z_{1}, z_{2}, z_{3}\right) \longmapsto\left(-z_{1}, i z_{2}, i z_{3}\right) .
$$

Define $G=\left\{1, \kappa, \kappa^{2}, \kappa^{3}\right\}$, so that $G$ is a finite subgroup of $S U(3)$ isomorphic to $\mathbb{Z}_{4}$. The only fixed point of $\kappa$ and $\kappa^{3}$ is $(0,0,0)$, but the fixed points of $\kappa^{2}$ are $\left(z_{1}, 0,0\right)$ for all $z_{1} \in \mathbb{C}$. Therefore $\mathbb{C}^{3} / G$ has singularities of codimension two. The subgroup of $G$ fixing the points $\left(z_{1}, 0,0\right)$ is $H=\left\{1, \kappa^{2}\right\}$, which is a normal subgroup of $G$, and preserves the obvious splitting $\mathbb{C}^{3}=\mathbb{C} \oplus \mathbb{C}^{2}$. Thus the theory of $\S 3$ applies to $G$ and $H$.

The Kleinian singularity $\mathbb{C}^{2} / H$ is $\mathbb{C}^{2} /\{ \pm 1\}$. The crepant resolution $X$ of $\mathbb{C}^{2} /\{ \pm 1\}$ has $H_{2}(X, \mathbb{Z})=\mathbb{Z}$. The Dynkin diagram $\Gamma$ is $A_{1}$, with $\operatorname{Aut}(\Gamma)=\{1\}$ and Weyl group $W=\mathbb{Z}_{2}$. Let $W=\{1, \lambda\}$. Then the generator $\lambda$ of $W$ acts on $H_{2}(X, \mathbb{Z})$ by multiplication by -1 . The quotient group $K=G / H$ is $\mathbb{Z}_{2}$ with generator $\kappa H$. Thus the homomorphism $\chi: K \rightarrow \operatorname{Aut}(\Gamma) \ltimes W$ of $\S 3$ maps $\mathbb{Z}_{2}$ to $\mathbb{Z}_{2}$, and the condition $\pi \circ \chi=\psi$ on $\chi$ is trivial since $\operatorname{Aut}(\Gamma)=\{1\}$. Therefore there are two possibilities for $\chi$, given by

$$
\text { (a) } \chi(H)=1, \chi(\kappa H)=1, \quad \text { and } \quad(b) \chi(H)=1, \chi(\kappa H)=\lambda .
$$

Since $H^{2}(X, \mathbb{R}) \cong \mathbb{R}$ and $H^{2}(X, \mathbb{C}) \cong \mathbb{C}$, the ALE spaces asymptotic to $\mathbb{C}^{2} / H$ are parametrized by pairs $(\alpha, \beta)$ with $\alpha \in \mathbb{R}$ and $\beta \in \mathbb{C}$. The condition for $(\alpha, \beta) \in U$ is that either $\alpha \neq 0$ or $\beta \neq 0$. Let us calculate the conditions $(3.1)$ on $(\alpha, \beta)$ for the ALE space $X_{\alpha, \beta}$ to admit a suitable $K$-action, for each possibility (a) and (b) in (4.2). These conditions involve $\phi$, which is given by $\phi(H)=1, \phi(\kappa H)=-1$. In case (a), with $g H=\kappa H$, equation (3.1) gives $\alpha=\alpha$ and $\beta=-\beta$, which holds if $\beta=0$. In case (b) with $g H=\kappa H$, equation (3.1) gives $\alpha=-\alpha$ and $\beta=\beta$, which holds if $\alpha=0$.

Thus, section 3 gives two different ways (a) and (b) to choose an ALE space $X_{\alpha, \beta}$ asymptotic to $\mathbb{C}^{2} /\{ \pm 1\}$ together with a $K$-action on $\mathbb{C} \times X_{\alpha, \beta}$ asymptotic to the given action of $K$ on $\mathbb{C}^{3} / H$. Here is what happens in each case.

(a) Let $\alpha \in \mathbb{R}$ be nonzero. Then $K$ acts on $\mathbb{C} \times X_{\alpha, 0}$. The fixed points of $\kappa H$ in $\mathbb{C} \times X_{\alpha, 0}$ are a copy of $\mathbb{C P}^{1}$. Thus $\left(\mathbb{C} \times X_{\alpha, 0}\right) / K$ has singularities in codimension two. These singularities admit no deformations, but they do 
have a unique crepant resolution $Y_{1}$, which can be described explicitly using toric geometry, and is a crepant resolution of $\mathbb{C}^{3} / \mathbb{Z}_{4}$. The Betti numbers $b^{j}=b^{j}\left(Y_{1}\right)$ of $Y_{1}$ are

$$
b^{0}=b^{4}=1, \quad b^{2}=2, \quad b^{1}=b^{3}=b^{5}=b^{6}=0 .
$$

(b) Let $\beta \in \mathbb{C}$ be nonzero. Then $X_{0, \beta}$ is isomorphic as a complex surface to the hypersurface $x_{1} x_{3}-x_{2}^{2}=\beta$ in $\mathbb{C}^{3}$. Using these coordinates on $X_{0, \beta}$, the $K$-action on $\mathbb{C} \times X_{0, \beta}$ is given by $\kappa H \cdot\left(z_{1}, x_{1}, x_{2}, x_{3}\right)=\left(-z_{1},-x_{1},-x_{2},-x_{3}\right)$. Now this action has no fixed points in $\mathbb{C} \times X_{0, \beta}$, since $(0,0,0,0)$ does not satisfy $x_{1} x_{3}-x_{2}^{2}=\beta$. Thus $Y_{2}=\left(\mathbb{C} \times X_{0, \beta}\right) / K$ is already nonsingular, and is a smoothing of $\mathbb{C}^{3} / \mathbb{Z}_{4}$.

A careful analysis shows that $Y_{2}$ retracts onto the subset

$$
\left\{ \pm\left(0, x_{1}, x_{2}, x_{3}\right) \in Y_{2}:\left|x_{1}\right|^{2}+2\left|x_{2}\right|^{2}+\left|x_{3}\right|^{2}=2|\beta|\right\},
$$

which is a copy of $\mathbb{R P}^{2}$. Thus the fundamental group and cohomology of $Y_{2}$ and $\mathbb{R P}^{2}$ are isomorphic. So $\pi_{1}\left(Y_{2}\right) \cong \mathbb{Z}_{2}$, and the Betti numbers $b^{j}=b^{j}\left(Y_{2}\right)$ are

$$
b^{0}=1, \quad b^{1}=\cdots=b^{6}=0 .
$$

From (4.3) and (4.4) we see that methods (a) and (b) yield a crepant resolution $Y_{1}$ and a smoothing $Y_{2}$ of $\mathbb{C}^{3} / \mathbb{Z}_{4}$ with rather different topology. The reason for this difference is that in case (a), $\kappa H$ acts trivially on $H_{2}\left(X_{\alpha, 0}, \mathbb{Z}\right)$, but in case (b), $\kappa H$ acts on $H_{2}\left(X_{0, \beta}, \mathbb{Z}\right)$ by multiplication by -1 , so the two $K$-actions are topologically distinct.

4.2. An orbifold $T^{6} / \mathbb{Z}_{4}$ and how to desingularize it. Let $\mathbb{C}^{3}$ have complex coordinates $\left(z_{1}, z_{2}, z_{3}\right)$, and define a lattice $\Lambda$ in $\mathbb{C}^{3}$ by

$$
\Lambda=\left\{\left(a_{1}+i b_{1}, a_{2}+i b_{2}, a_{3}+i b_{3}\right): a_{j}, b_{j} \in \mathbb{Z}\right\} .
$$

Then $\mathbb{C}^{3} / \Lambda$ is a 6 -torus $T^{6}$, equipped with a flat Calabi-Yau structure. Let $\kappa$ act on $T^{6}$ by

$$
\kappa:\left(z_{1}, z_{2}, z_{3}\right)+\Lambda \longmapsto\left(-z_{1}, i z_{2}, i z_{3}\right)+\Lambda,
$$

as in (4.1). Then $\kappa$ is well-defined and preserves the Calabi-Yau structure on $T^{6}$. Let $G=\left\{1, \kappa, \kappa^{2}, \kappa^{3}\right\}$ be the group generated by $\kappa$, so that $G \cong \mathbb{Z}_{4}$. Then $T^{6} / G$ is a compact Calabi-Yau orbifold. To understand the singular set of $T^{6} / G$, we shall first find the fixed points of $\kappa, \kappa^{2}$ and $\kappa^{3}$.

The subset of $T^{6}$ fixed by $\kappa$ and $\kappa^{3}$ turns out to be the 16 points

$$
\left\{\left(z_{1}, z_{2}, z_{3}\right)+\Lambda: z_{1} \in\left\{0, \frac{1}{2}, \frac{1}{2} i, \frac{1}{2}+\frac{1}{2} i\right\}, z_{2}, z_{3} \in\left\{0, \frac{1}{2}+\frac{1}{2} i\right\}\right\} .
$$

And the subset of $T^{6}$ fixed by $\kappa^{2}$ is 16 copies of $T^{2}$, given by

$$
\left\{\left(z_{1}, z_{2}, z_{3}\right)+\Lambda: z_{1} \in \mathbb{C}, z_{2}, z_{3} \in\left\{0, \frac{1}{2}, \frac{1}{2} i, \frac{1}{2}+\frac{1}{2} i\right\}\right\} .
$$

Twelve of the 16 copies of $T^{2}$ fixed by $\kappa^{2}$ are identified in pairs by the action of $\kappa$, and these contribute 6 copies of $T^{2}$ to the singular set of $T^{6} / G$. On the remaining 4 copies $\kappa$ acts as -1 , so these contribute 4 copies of $T^{2} /\{ \pm 1\}$ to the singular set. Each $T^{2} /\{ \pm 1\}$ contains 4 of the 16 points fixed by $\kappa$. 
Therefore the singular set of $T^{6} / G$ consists of 6 copies of $T^{2}$, with singularities modelled on $T^{2} \times \mathbb{C}^{2} /\{ \pm 1\}$, and 4 copies of $T^{2} /\{ \pm 1\}$. Each $T^{2} /\{ \pm 1\}$ has 4 special points where the singularity is modelled on 0 in $\mathbb{C}^{3} / G$ as in $\S 4.1$, and the other singular points look locally like the singularities of $\mathbb{C} \times \mathbb{C}^{2} /\{ \pm 1\}$.

To desingularize $T^{6} / G$, each copy of $T^{2}$ in the singular set can be resolved with a crepant resolution, which replaces the $T^{2}$ by $T^{2} \times \mathbb{C P}^{1}$. But each copy of $T^{2} /\{ \pm 1\}$ in the singular set, may be either resolved using method (a) or smoothed using method (b) above. For each $k=0, \ldots, 4$, let $Z_{k}$ be one of the manifolds obtained by resolving each $T^{2}$ in the singular set, resolving using method (a) for $k$ of the $T^{2} /\{ \pm 1\}$ 's, and smoothing using method (b) for the remaining $4-k$ copies of $T^{2} /\{ \pm 1\}$. Then each $Z_{k}$ is a compact, nonsingular manifold carrying a family of Calabi-Yau structures.

We shall find the Betti numbers of $Z_{k}$. The Betti numbers of $T^{6} / G$ are

$$
b^{0}\left(T^{6} / \mathbb{Z}_{4}\right)=1, \quad b^{1}\left(T^{6} / \mathbb{Z}_{4}\right)=0, \quad b^{2}\left(T^{6} / \mathbb{Z}_{4}\right)=5, \quad b^{3}\left(T^{6} / \mathbb{Z}_{4}\right)=4 .
$$

To find the Betti numbers of $Z_{k}$ we must add on contributions from each component of the singular set. The resolution of each copy of $T^{2}$ in the singular set adds 1 to $b^{2}$ and 2 to $b^{3}$. Resolving a $T^{2} /\{ \pm 1\}$ using method (a) adds 5 to $b^{2}$ and fixes $b^{3}$, but smoothing using method (b) fixes $b^{2}$ and adds 2 to $b^{3}$. All three processes fix $b^{0}$ and $b^{1}$.

Thus we calculate that the Calabi-Yau 3-folds $Z_{k}$ have Betti numbers

$$
b^{0}\left(Z_{k}\right)=1, \quad b^{1}\left(Z_{k}\right)=0, \quad b^{2}\left(Z_{k}\right)=11+5 k, \quad b^{3}\left(Z_{k}\right)=24-2 k,
$$

giving Euler characteristic $\chi\left(Z_{k}\right)=12 k$. For $k=1,2,3,4$ one can show that $Z_{k}$ is simply-connected, and carries metrics with holonomy $S U(3)$. But $Z_{0}$ is the quotient of $T^{2} \times K 3$ by a free $\mathbb{Z}_{2}$-action, and has fundamental group $\mathbb{Z}_{2} \ltimes \mathbb{Z}^{2}$ and holonomy $\mathbb{Z}_{2} \times$ $S U(2)$.

5. Another example. In this section we consider another example that shows how complicated the business of desingularizing orbifolds can be. First in $\S 5.1$ we describe the crepant resolutions, smoothings and CR-deformations of $\mathbb{C}^{3} / \mathbb{Z}_{2}^{2}$. Then in $§ 5.2$ we apply this to study the possible desingularizations of the orbifold $T^{6} / \mathbb{Z}_{2}^{2}$. This turns out to be a complex problem, which we do not solve completely.

5.1. Desingularizations of $\mathbb{C}^{3} / \mathbb{Z}_{2}^{2}$. Let $\mathbb{C}^{3}$ have complex coordinates $\left(z_{1}, z_{2}, z_{3}\right)$, and define $\kappa_{j}: \mathbb{C}^{3} \rightarrow \mathbb{C}^{3}$ by

$$
\begin{aligned}
\kappa_{1}:\left(z_{1}, z_{2}, z_{3}\right) \mapsto\left(z_{1},-z_{2},-z_{3}\right), & \kappa_{2}:\left(z_{1}, z_{2}, z_{3}\right) \mapsto\left(-z_{1}, z_{2},-z_{3}\right) \\
\text { and } \quad \kappa_{3}:\left(z_{1}, z_{2}, z_{3}\right) & \mapsto\left(-z_{1},-z_{2}, z_{3}\right) .
\end{aligned}
$$

Then $G=\left\{1, \kappa_{1}, \kappa_{2}, \kappa_{3}\right\}$ is a subgroup of $S U(3)$ isomorphic to $\mathbb{Z}_{2}^{2}$. The singular set of $\mathbb{C}^{3} / G$ splits into 3 pieces: the points $\pm\left(z_{1}, 0,0\right)$ coming from the fixed points of $\kappa_{1}$, the points $\pm\left(0, z_{2}, 0\right)$ from the fixed points of $\kappa_{2}$, and the points $\pm\left(0,0, z_{3}\right)$ from the fixed points of $\kappa_{3}$. Each piece is a copy of $\mathbb{C} /\{ \pm 1\}$, and they meet at $(0,0,0)$.

Define $H_{j}=\left\{1, \kappa_{j}\right\}$ for $j=1,2,3$. Then $H_{1}, H_{2}$ and $H_{3}$ are normal subgroups of $G$ with $\mathbb{C}^{3} / H_{j} \cong \mathbb{C} \times \mathbb{C}^{2} /\{ \pm 1\}$. The quotient groups $K_{j}=G / H_{j}$ are isomorphic to $\mathbb{Z}_{2}$, and act upon $\mathbb{C}^{3} / H_{j}$. Thus we can apply the method of $\S 3$ to $\mathbb{C}^{3} / G$ in 3 different ways, by starting with $H_{1}, H_{2}$ or $H_{3}$, and these 3 ways correspond to the 3 pieces of the singular set. Now $\mathbb{C}^{2} /\{ \pm 1\}$ has Dynkin diagram $\Gamma=A_{1}$, with $\operatorname{Aut}(\Gamma)=\{1\}$ and Weyl group $W=\{1, \lambda\}$ isomorphic to $\mathbb{Z}_{2}$. 
Thus, by $\S 3$, every desingularization $Y$ of $\mathbb{C}^{3} / \mathbb{Z}_{2}^{2}$ has three pieces of topological data, the group homomorphisms $\chi_{j}: K_{j} \rightarrow \operatorname{Aut}(\Gamma) \ltimes W$ for $j=1,2,3$. Here $K_{1}=$ $\left\{H_{1}, \kappa_{2} H_{1}\right\}$ and $\operatorname{Aut}(\Gamma) \ltimes W=\{1, \lambda\}$ are both isomorphic to $\mathbb{Z}_{2}$, so there are two possibilities for $\chi_{1}$,

$$
\text { (a) } \chi_{1}\left(H_{1}\right)=1, \chi_{1}\left(\kappa_{2} H_{1}\right)=1, \quad \text { and } \quad(b) \chi_{1}\left(H_{1}\right)=1, \chi_{1}\left(\kappa_{2} H_{1}\right)=\lambda .
$$

As a shorthand, we shall write $\chi_{1}=1$ to denote case (a) and $\chi_{1}=-1$ to denote case (b). Similarly, there are two possibilities for each of $\chi_{2}$ and $\chi_{3}$, which we will also write $\chi_{2}= \pm 1, \chi_{3}= \pm 1$. We can think of $\chi_{1}, \chi_{2}$ and $\chi_{3}$ as describing the topology of $Y$ near infinity.

First we describe the deformations of $\mathbb{C}^{3} / \mathbb{Z}_{2}^{2}$. Let $\gamma: \mathbb{C}^{3} / \mathbb{Z}_{2}^{2} \rightarrow \mathbb{C}^{4}$ be given by $\gamma\left(\left(z_{1}, z_{2}, z_{3}\right) G\right)=\left(z_{1}^{2}, z_{2}^{2}, z_{3}^{2}, z_{1} z_{2} z_{3}\right)$. Then $\gamma$ is well-defined, and induces an isomorphism between $\mathbb{C}^{3} / G$ and the hypersurface

$$
W_{0,0,0,0}=\left\{\left(x_{1}, x_{2}, x_{3}, x_{4}\right) \in \mathbb{C}^{4}: x_{1} x_{2} x_{3}-x_{4}^{2}=0\right\}
$$

in $\mathbb{C}^{4}$. Let $\alpha, \beta_{1}, \beta_{2}$ and $\beta_{3}$ be complex numbers, and define

$$
W_{\alpha, \beta_{1}, \beta_{2}, \beta_{3}}=\left\{\left(x_{1}, x_{2}, x_{3}, x_{4}\right) \in \mathbb{C}^{4}: x_{1} x_{2} x_{3}-x_{4}^{2}=\alpha+\beta_{1} x_{1}+\beta_{2} x_{2}+\beta_{3} x_{3}\right\} .
$$

Then $W_{\alpha, \beta_{1}, \beta_{2}, \beta_{3}}$ is a deformation of $\mathbb{C}^{3} / \mathbb{Z}_{2}^{2}$. For generic $\alpha, \ldots, \beta_{3}$ the hypersurface $W_{\alpha, \beta_{1}, \beta_{2}, \beta_{3}}$ is nonsingular, and thus is a smoothing of $\mathbb{C}^{3} / \mathbb{Z}_{2}^{2}$. But for some special values of $\alpha, \ldots, \beta_{3}$ it has singularities, and we then take a crepant resolution to get a CR-deformation of $\mathbb{C}^{3} / \mathbb{Z}_{2}^{2}$.

We will now list the different cases that arise in this way. In each case we will give the values of $\chi_{1}, \chi_{2}$ and $\chi_{3}$, and the Betti numbers $b^{2}$ and $b^{3}$ of the desingularization.

(i) $W_{0,0,0,0}$ is isomorphic to $\mathbb{C}^{3} / \mathbb{Z}_{2}^{2}$. It has 4 possible crepant resolutions, which are easily described using toric geometry. Each has $\chi_{1}=\chi_{2}=\chi_{3}=1$ and Betti numbers $b^{2}=3$ and $b^{3}=0$, and is a crepant resolution of $\mathbb{C}^{3} / \mathbb{Z}_{2}^{2}$.

(ii) $W_{\alpha, 0,0,0}$ for $\alpha \neq 0$. This is nonsingular, with $\chi_{1}=\chi_{2}=\chi_{3}=1, b^{2}=2$ and $b^{3}=1$, and is a smoothing of $\mathbb{C}^{3} / \mathbb{Z}_{2}^{2}$.

(iii) $W_{0, \beta_{1}, 0,0}$ for $\beta_{1} \neq 0$. This is isomorphic to $\left(\mathbb{C} \times X_{0, \beta_{1}}\right) / K_{1}$, and has singularities at the points $\left(0, x_{2}, x_{3}, 0\right)$ for $x_{2} x_{3}=\beta_{1}$. It has a unique crepant resolution, by blowing up the singular set, which has $\chi_{1}=-1, \chi_{2}=\chi_{3}=1$ and $b^{2}=b^{3}=1$, and is a CR-deformation of $\mathbb{C}^{3} / \mathbb{Z}_{2}^{2}$.

(iv) $W_{\alpha, \beta_{1}, 0,0}$ for $\alpha, \beta_{1} \neq 0$. This is nonsingular, and thus a smoothing of $\mathbb{C}^{3} / \mathbb{Z}_{2}^{2}$. It is a smooth deformation of the 3 -fold in (iii), with the same topology and values of $\chi_{j}$ and $b^{k}$.

(v),(vi) As (iii) and (iv) but with $\beta_{2}$ nonzero and $\chi_{2}=-1$, instead of $\beta_{1}$ and $\chi_{1}$.

(vii),(viii) As (iii) and (iv) but with $\beta_{3}$ nonzero and $\chi_{3}=-1$, instead of $\beta_{1}$ and $\chi_{1}$.

(ix) $W_{\alpha, \beta_{1}, \beta_{2}, \beta_{3}}$ with $\beta_{1}, \beta_{2}, \beta_{3} \neq 0$ and $\alpha^{2} \neq 4 \beta_{1} \beta_{2} \beta_{3}$. This is nonsingular and has $\chi_{1}=\chi_{2}=\chi_{3}=-1, b^{2}=0$ and $b^{3}=2$, and is a smoothing of $\mathbb{C}^{3} / \mathbb{Z}_{2}^{2}$.

For a few special values of $\alpha, \ldots, \beta_{3}$, we cannot resolve $W_{\alpha, \beta_{1}, \beta_{2}, \beta_{3}}$ as a CalabiYau 3-fold with the appropriate asymptotic behaviour, so it does not appear on the above list. Here are the missing cases, with the reason why.

- If exactly one of $\beta_{1}, \beta_{2}, \beta_{3}$ is zero, say $\beta_{1}$, then $W_{\alpha, 0, \beta_{2}, \beta_{3}}$ is nonsingular and is topologically equivalent to case (ix). However, we should regard it as being 'singular at infinity'.

- Also, $W_{\alpha, \beta_{1}, \beta_{2}, \beta_{3}}$ with $\beta_{1}, \beta_{2}, \beta_{3} \neq 0$ and $\alpha^{2}=4 \beta_{1} \beta_{2} \beta_{3}$ has a single node at $x_{j}=-\alpha / 2 \beta_{j}$ for $j=1,2,3$. But neither of the small resolutions of it are Kähler manifolds. 
Here is what we mean by 'singular at infinity'. Our goal is to construct CalabiYau 3-folds that desingularize $\mathbb{C}^{3} / \mathbb{Z}_{2}^{2}$. As with the ALE spaces of $\S 2$, we expect these manifolds to be asymptotic to $\mathbb{C}^{3} / \mathbb{Z}_{2}^{2}$ at infinity, and the metrics on them to be asymptotic at infinity to the Euclidean metric on $\mathbb{C}^{3} / \mathbb{Z}_{2}^{2}$, in some suitable sense. However, because the singularities of $\mathbb{C}^{3} / \mathbb{Z}_{2}^{2}$ extend to infinity, things are more complicated than they seem at first.

In [4], the author studies Kähler metrics on resolutions $X$ of non-isolated quotient singularities $\mathbb{C}^{m} / G$, under appropriate asymptotic conditions. We define Quasi-ALE metrics upon $X$, which appear to be the natural generalization of ALE metrics to the non-isolated case, and prove an existence result for Ricci-flat Quasi-ALE Kähler metrics on crepant resolutions of $\mathbb{C}^{m} / G$. Further work will be published in the author's book [5], including an extension to the case of smoothings and CR-deformations of $\mathbb{C}^{m} / G$. In cases (i)-(ix) above, these results guarantee the existence of Calabi-Yau metrics on the given desingularizations $Y$, for suitable choices of the Kähler class.

Roughly speaking, in this case the asymptotic conditions on the metrics are as follows. If at least two of $z_{1}, z_{2}, z_{3}$ are very large, then the metric on $Y$ near the point $\left(z_{1}, z_{2}, z_{3}\right) G$ in $\mathbb{C}^{3} / \mathbb{Z}_{2}^{2}$ must be close to the flat metric on $\mathbb{C}^{3} / \mathbb{Z}_{2}^{2}$. But if only one of $z_{1}, z_{2}, z_{3}$ is large, say $z_{1}$, then the metric on $Y$ near $\left(z_{1}, z_{2}, z_{3}\right) G$ in $\mathbb{C}^{3} / \mathbb{Z}_{2}^{2}$ must be close to the product metric on $\mathbb{C} \times X_{\delta, \epsilon}$, where $z_{1}$ is the coordinate in $\mathbb{C}$, and $X_{\delta, \epsilon}$ is an ALE space asymptotic to $\mathbb{C}^{2} /\{ \pm 1\}$, which has coordinates $\pm\left(z_{2}, z_{3}\right)$.

We say the metric on $Y$ is singular at infinity if the ALE space $X_{\delta, \epsilon}$ appearing in this asymptotic condition is singular - in this case, if $X_{\delta, \epsilon}=\mathbb{C}^{2} /\{ \pm 1\}$. We have excluded cases like $W_{\alpha, 0, \beta_{2}, \beta_{3}}$ for $\beta_{2}, \beta_{3} \neq 0$ from our list because they are singular at infinity, so that the singularities $\pm\left(z_{1}, 0,0\right)$ for $z_{1}$ very large, effectively remain unresolved. It can be shown that every Calabi-Yau desingularization $Y$ of $\mathbb{C}^{3} / \mathbb{Z}_{2}^{2}$ that is not 'singular at infinity', is modelled on one of cases (i)-(ix) above.

In cases (ii), (iv), (vi) and (viii) there are nontrivial conditions upon the Kähler class for the metrics to be nonsingular at infinity. The allowed values for the Kähler class split into several connected components - six components in case (ii) and two components in cases (iv), (vi) and (viii). The connected component of the Kähler class can be regarded as an extra topological choice in the desingularization; but we will not discuss this issue here.

Observe that in cases (i)-(ix) we can have 0,1 or 3 of $\chi_{1}, \chi_{2}$ and $\chi_{3}$ equal to -1 , but we cannot have exactly 2 of $\chi_{1}, \chi_{2}$ and $\chi_{3}$ equal to -1 . Thus we cannot choose $\chi_{1}, \chi_{2}$ and $\chi_{3}$ independently. The moral is that when we desingularize $\mathbb{C}^{3} / \mathbb{Z}_{2}^{2}$ or other orbifolds in which the codimension 2 singularities split into several pieces, the topological choices for different pieces of the singular set are not in general independent, but are subject to constraints involving all the pieces.

5.2. Counting Calabi-Yau desingularizations of $T^{6} / \mathbb{Z}_{2}^{2}$. Let $\Lambda$ be as in $\S 4.2$, so that $\mathbb{C}^{3} / \Lambda$ is a 6 -torus $T^{6}$ with a flat Calabi-Yau structure. Let $\kappa_{1}, \kappa_{2}$ and $\kappa_{3}$ act on $T^{6}$ by

$$
\begin{aligned}
& \kappa_{1}:\left(z_{1}, z_{2}, z_{3}\right)+\Lambda \longmapsto\left(z_{1},-z_{2},-z_{3}\right)+\Lambda, \\
& \kappa_{2}:\left(z_{1}, z_{2}, z_{3}\right)+\Lambda \longmapsto\left(-z_{1}, z_{2},-z_{3}\right)+\Lambda,
\end{aligned}
$$

and $\kappa_{3}=\kappa_{1} \kappa_{2}$, as in (5.1). Then $G=\left\{1, \kappa_{1}, \kappa_{2}, \kappa_{3}\right\}$ acts on $T^{6}$ preserving the CalabiYau structure, and is a group isomorphic to $\mathbb{Z}_{2}^{2}$. The quotient $T^{6} / G$ is a Calabi-Yau orbifold, with singularities modelled on $\mathbb{C}^{3} / \mathbb{Z}_{2}^{2}$. This orbifold was studied by Vafa and Witten $[15, \S 2]$, who showed that $T^{6} / \mathbb{Z}_{2}^{2}$ has many crepant resolutions, which all have 
$h^{1,1}=51$ and $h^{2,1}=3$. They also found one smoothing of $T^{6} / \mathbb{Z}_{2}^{2}$, which has $h^{1,1}=3$ and $h^{2,1}=115$.

Let us now consider how to describe all the Calabi-Yau 3-folds constructed from $T^{6} / \mathbb{Z}_{2}^{2}$ by crepant resolutions, smoothings and CR-deformations. We will not be able to offer a complete classification, because the calculations involved are extremely complex. However, we can explain the first steps in this classification, and we will see that there are in fact a large number of possibilities, of which those found by Vafa and Witten represent two extremes.

We shall regard $T^{6}$ as a product $T^{2} \times T^{2} \times T^{2}$. Then $\mathbb{Z}_{2}$ acts on each copy of $T^{2}$, so that $\mathbb{Z}_{2}^{3}$ acts on $T^{6}$, and $G$ is a subgroup of this $\mathbb{Z}_{2}^{3}$. This $\mathbb{Z}_{2}$-action on $T^{2}$ has 4 fixed points $p_{1}, \ldots, p_{4}$. The fixed points of $\kappa_{1}$ on $T^{6}$ are $T^{2} \times p_{j} \times p_{k}$ for $j, k=1, \ldots, 4$, which is 16 copies of $T^{2}$. For $j, k=1, \ldots, 4$, define $A_{j k}=T^{2} / \mathbb{Z}_{2} \times p_{j} \times p_{k} \subset T^{6} / \mathbb{Z}_{2}^{2}$. Similarly, for $i, k=1, \ldots, 4$, define $B_{i k}=p_{i} \times T^{2} / \mathbb{Z}_{2} \times p_{k} \subset T^{6} / \mathbb{Z}_{2}^{2}$, and for $i, j=$ $1, \ldots, 4$ define $C_{i j}=p_{i} \times p_{j} \times T^{2} / \mathbb{Z}_{2} \subset T^{6} / \mathbb{Z}_{2}^{2}$.

The singular set of $T^{6} / \mathbb{Z}_{2}^{2}$ is the union of these sets $A_{j k}, B_{i k}$ and $C_{i j}$. The $A_{j k}$ come from the fixed points of $\kappa_{1}$, the $B_{i k}$ from $\kappa_{2}$, and the $C_{i j}$ from $\kappa_{3}$. Each of the $A_{j k}, B_{i k}$ and $C_{i j}$ is a copy of $T^{2} / \mathbb{Z}_{2}$. They are not disjoint, but for each $i, j, k=1, \ldots, 4$ the three sets $A_{j k}, B_{i k}$ and $C_{i j}$ intersect in the point $p_{i j k}=p_{i} \times p_{j} \times p_{k}$ in $T^{6} / \mathbb{Z}_{2}^{2}$. The $p_{i j k}$ are the 64 singular points in $T^{6} / \mathbb{Z}_{2}^{2}$ which have a singularity modelled on 0 in $\mathbb{C}^{3} / \mathbb{Z}_{2}^{2}$.

Now, following the method of $\S 3$, to desingularize $T^{6} / \mathbb{Z}_{2}^{2}$ we must first choose some topological data, the group homomorphism $\chi$. As we saw above, for the $\mathbb{C}^{3} / \mathbb{Z}_{2}^{2}$ singularity there are 3 pieces of data $\chi_{1}, \chi_{2}, \chi_{3}$ corresponding to the $\kappa_{1}, \kappa_{2}$ and $\kappa_{3}$ singularities, and each $\chi_{j}$ can take the values \pm 1 . In our case, a little thought shows that $\chi_{1}$ gives topological information about the way the singularities $A_{j k}$ are resolved. One can show that $\chi_{1}$ must be constant on each $A_{j k}$, since $A_{j k}$ is connected, but different $A_{j k}$ can have different values of $\chi_{1}$. Write $\chi_{1, j k}$ for the value of $\chi_{1}$ on $A_{j k}$. Then for $j, k=1, \ldots, 4$, we have $\chi_{1, j k}= \pm 1$.

Similarly, $\chi_{2}$ gives information on how the $B_{i k}$ are resolved, and we write $\chi_{2, i k}$ for the value of $\chi_{2}$ on $B_{i k}$, and $\chi_{3}$ gives information on how the $C_{i j}$ are resolved, and we write $\chi_{3, i j}$ for the value of $\chi_{3}$ on $C_{i j}$. Thus, to desingularize $T^{6} / \mathbb{Z}_{2}^{2}$ we must first choose the values of $\chi_{1, j k}, \chi_{2, i k}$ and $\chi_{3, i j}$. These are 48 variables taking the values \pm 1 , so there are $2^{48}$, or about $2 \cdot 8 \times 10^{14}$ possible choices.

Now, we saw above that cases (i)-(ix) allow 0,1 or 3 of $\chi_{1}, \chi_{2}, \chi_{3}$ to be -1 , but not two to be -1 . This condition applies at each of the 64 points $p_{i j k}$. Therefore, a necessary condition for the data $\chi_{i, j k}$ to represent a possible Calabi-Yau 3-fold is that for each set of values $i, j, k=1, \ldots, 4$, exactly 0,1 or 3 of $\chi_{1, j k}, \chi_{2, i k}$ and $\chi_{3, i j}$ are -1 , but not two of them.

This condition excludes nearly all of the $2^{48}$ choices for the $\chi_{i, j k}$, but there are still many choices for which this condition is satisfied, although we have not been able to count them. However, we can give four explicit families of solutions to the conditions, and so find a lower limit for their number. For the first family, let $\delta_{i}, \epsilon_{j}$ and $\zeta_{k}$ take the values \pm 1 for $i, j, k=1, \ldots, 4$, and define

$$
\chi_{1, j k}=\epsilon_{j} \zeta_{k}, \quad \chi_{2, i k}=\delta_{i} \zeta_{k} \quad \text { and } \quad \chi_{3, i j}=-\delta_{i} \epsilon_{j} .
$$

Then $\chi_{1, j k} \chi_{2, i k} \chi_{3, i j}=-1$ for all $i, j, k$, and this means that either 1 or 3 of $\chi_{1, j k}, \chi_{2, i k}$ and $\chi_{3, i j}$ are equal to -1 , but not 0 or 2 . Conversely, any set of values of $\chi_{1, j k}, \chi_{2, i k}$ and $\chi_{3, i j}$ for which this holds may be written in the form (5.2). There are $2^{12}$ possible values for $\delta_{i}, \epsilon_{j}$ and $\zeta_{k}$, but reversing the sign of all the $\delta_{i}, \epsilon_{j}$ and $\zeta_{k}$ does not change the $\chi_{i, j k}$, so this gives $2^{11}=2048$ different solutions for the $\chi_{i, j k}$. 
For the second family, let $\chi_{2, i k}=\chi_{3, i j}=1$ for all $i, j, k$, and let $\chi_{1, j k}$ be \pm 1 . Clearly, for all $i, j, k$ either 0 or 1 of $\chi_{1, j k}, \chi_{2, i k}$ and $\chi_{3, i j}$ are equal to -1 , so the condition is satisfied. There are $2^{16}=65536$ possible choices for the $\chi_{1, j k}$. Similarly, by putting $\chi_{1, j k}=\chi_{3, i j}=1$, and by putting $\chi_{1, j k}=\chi_{2, i k}=1$, we get two other families of $2^{16}$ choices. In total, allowing for repeated choices, we have found 198651 different sets of values for $\chi_{1, j k}, \chi_{2, i k}$ and $\chi_{3, i j}$ in which the conditions are satisfied. This is a lower limit on the number of solutions, which is probably rather larger than this.

Next, having chosen a set of suitable values of the $\chi_{i, j k}$, we must look for crepant resolutions, smoothings and CR-deformations of $T^{6} / \mathbb{Z}_{2}^{2}$ with this data. There are still further topological choices to make. At each of the 64 points $p_{i j k}$, we must choose one of (i)-(ix) above that is consistent with the values of $\chi_{1, j k}, \chi_{2, i k}$ and $\chi_{3, i j}$ already chosen. For instance, if $\chi_{1, j k}=\chi_{2, i k}=\chi_{3, i j}=1$ then either case (i) or case (ii) will do. There are also more subtle topological choices to do with Weyl groups and the connected component of the Kähler class, which we will not go into.

Having made all these topological choices, we can finally construct a unique real 6-manifold $Y$ that desingularizes $T^{6} / \mathbb{Z}_{2}^{2}$, which locally has the topology of a CalabiYau desingularization. However, many of these 6-manifolds do not admit Calabi-Yau structures desingularizing $T^{6} / \mathbb{Z}_{2}^{2}$, for reasons of global topology. For $Y$ to be Kähler we must be able to choose a Kähler class $[\omega]$ in $H^{2}(Y, \mathbb{R})$ that is positive on all the complex curves in $Y$ introduced by the crepant resolutions, and this is not always possible. Similar conditions apply to the choice of $[\Omega]$ in $H^{3}(Y, \mathbb{C})$.

But in some special cases we can see quite easily that the Calabi-Yau structures exist. For instance, in the second family above with $\chi_{2, i k}=\chi_{3, i j}=1$, if we choose desingularization (i) for $p_{i j k}$ when $\chi_{1, j k}=1$ and desingularization (iii) for $p_{i j k}$ when $\chi_{1, j k}=-1$, then one can prove that the resulting manifold has a Calabi-Yau structure, which is a crepant resolution of $\left(T^{2} \times K 3\right) / \mathbb{Z}_{2}$. Using the same trick with the third and fourth families gives a total of 196606 different sets of values of the $\chi_{i, j k}$ which do correspond to Calabi-Yau 3-folds; and as there are four topological choices for resolution (i), these will lead to many more manifolds.

Our discussion has shown that the problem of classifying all the possible CalabiYau desingularizations of $T^{6} / \mathbb{Z}_{2}^{2}$ is of great complexity. There are a large number of choices to be made, but these choices are subject to many complicated conditions. The author's feeling is that these conditions are not too restrictive, and the number of different crepant resolutions, smoothings and CR-deformations of $T^{6} / \mathbb{Z}_{2}^{2}$ is probably very large.

\section{REFERENCES}

[1] V. I. ARNOLD, Some remarks on symplectic monodromy of Milnor fibrations, in The Floer memorial volume, H. Hofer, C. Taubes, A. Weinstein and E. Zehnder, ed., Progress in Mathematics 133, Birkhäuser, 1995, pp. 99-104.

[2] P. Candelas, M. Lynker, And R. Schrimmrigk, Calabi-Yau manifolds in weighted $\mathbb{P}_{*}^{4}$, Nucl. Phys., B341 (1990), pp. 383-402.

[3] Y. ITO AND M. REID, The McKay correspondence for finite subgroups of $S L(3, \mathbb{C})$, Duke server alg-geom/9411010, 1994.

[4] D. D. JoYcE, Quasi-ALE metrics with holonomy $S U(m)$ and $S p(m)$, math.AG/9905043, 1999.

[5] D. D. JoYCE, Compact manifolds with special holonomy, to be published by Oxford University Press, 2000.

[6] P. B. Kronheimer, The construction of ALE spaces as hyperkähler quotients, J. Diff. Geom., 29 (1989), pp. 665-683. 
[7] P. B. Kronheimer, A Torelli-type theorem for gravitational instantons, J. Diff. Geom., 29 (1989), pp. 685-697.

[8] J. MCKAY, Graphs, singularities, and finite groups, in Proc. Symp. Pure Math. 37, A.M.S, 1980, pp. 183-186.

[9] M. REID, McKay correspondence, Duke server alg-geom/9702016, 1997.

[10] S.-S. RoAN, Minimal resolution of Gorenstein orbifolds, Topology, 35 (1996), pp. 489-508.

[11] S.-S. Roan And S.-T. YAU, On Ricci-flat 3-fold, Acta Math. Sinica, 3 (1987), pp. 256-288.

[12] P. SEIDEL, Symplectic automorphisms of $T^{*} S^{2}$, math. DG/9803084, 1998.

[13] P. Slodowy, Simple singularities and simple algebraic groups, Springer Lecture Notes in Math. $815,1980$.

[14] G. TIAN, Smoothing 3-folds with trivial canonical bundle and ordinary double points, in Mirror Symmetry I, S.-T. Yau, ed., International Press, 1998, pp. 399-420.

[15] C. VAfa And E. Witten, On orbifolds with discrete torsion, J. Geom. Phys., 15 (1995), pp. 189-214. 
D. JOYCE 